\title{
Dolor abdominal y parásitos intestinales en niños
}

Es conducta regular que ante todo niño con dolor abdominal en nuestros países en vía de desarrollo, el parasitismo intestinal sea tenido en cuenta. En este numero de la Revista Gastrohnup se presenta un artículo sobre Dolor abdominal en niños ${ }^{1}$ en donde se describe la fisiopatología del dolor abdominal así como semiológicamente como diferenciarlo en agudo, crónico y funcional, y dentro de estos, cuál es el papel que juegan los parásitos intestinales. En un segundo artículo de este número se realiza una revisión epidemiológica de un geohelmilto causante de dolor abdominal, el Ascaris lumbricoides ${ }^{2}$ y dentro del mismo su posible asociación con la presencia de dolor abdominal en niños.

Finalmente hay un par de artículos sobre nutrición pediátrica: el primero de ellos sobre la alimentación normal en el lactante normal3 y el segundo sobre nutrición en el niño con enfermedad renal.
El resto del numero de la Revista Gastrohnup está dedicado a una diversidad de artículos resultado de la productividad académica de algunos de los profesores de la Facultad de Salud de la Universidad del Valle.

'Ortíz-Rivera CJ. Dolor abdominal en niños. Rev Gastrohnup 2016; 18 (1): 32-36

${ }^{2}$ Grijalva-Zuluaga DS. Epidemiología del Áscaris lumbricoides en pediatría. Rev Gastrohnup 2016; 18 (1): $37-42$

CARLOS ALBERTO VELASCO-BENÍTEZ, MD Pediatra. Gastroenterólogo y nutriólogo Profesor Titular Departamento de Pediatría Universidad del Valle Cali, Colombia

Cali, abril 15 de 2016 